\title{
Question Answering System for an Effective Collaborative Learning
}

\author{
Prof. Kohei Arai ${ }^{1}$ \\ Information Science, \\ Saga University - Japan
}

\author{
Anik Nur Handayani ${ }^{1,2}$ \\ Electrical and Information Technology \\ State University of Malang - Indonesia
}

\begin{abstract}
The increasing advances of Internet Technologies in all application domains have changed life styles and interactions. With the rapid development of E-Learning, collaborative learning is an important for teaching, learning methods and strategies. Interaction between the students also student with the teacher is important for student to gain knowledge. Based on the four basic teaching styles formal authority, demonstrator or personal model, facilitator and delegator, today combined between facilitator and delegator style is responsible for student learning. It is student centered and the teacher as facilitates the material and activities, but learning becomes part of valuable and effective when they collaborate with each other, and as the teacher who will delegates and facilitates the responsibility of learning to the students. In this paper, we introduce an effective question answering Q\&A system for collaborative learning, which can act not just like a virtual teacher, but also virtual discussion for student. With the proposed system, brings a new Q\&A system, student can attach their question when they want collaborate using collaborative learning capitalize on one another's resources and skills. Students can ask their questions to the group when they want to collaborate with others, asking one another for information, evaluating one another's ideas, then each of the answer will compare with encyclopedia data base. In this research, the Q\&A system for the Senior High School in Indonesia, in this subject of Information Communication Technology implemented. From the the 40 question and 120 answer, the result is $\mathbf{9 0 , 4 8 \%}$ precision $\mathbf{5 0 \%}$ recall.
\end{abstract}

Keywords - component; E-Learning; Collaborative Learning; Q\&A; Knowledge Base.

\section{INTRODUCTION}

The concept of Collaborative Learning is two or more people learn or attempt to learn something together than independent. Different with individual learning, in collaborative learning people can exploit and share their resources and skills by asking for information, evaluating, monitoring one another's information and idea, etc [1]. Collaborative Learning is a model that knowledge can be created by sharing experiences within a population where members actively interact [2] [3]. In the Collaborative Robotic Instruction (A Graph Teaching Experience Computers and Education), the goal of collaborative learning is methodologies and environments which learners or students where each depends on and is responsible to each other [4]. Including both directly with face-to-face conversations [5] or using computer discussions (online forums, chat rooms, etc.) [6].

In [3] authors indicate that when they found some problem, students learn better when they learn together more frequently than working individually as members in a group. Indeed, the effectiveness of collaborative learning on the internet has been identified by various studies. Interaction among learners is fostered as communication over the internet is unpretentious and convenient when addressing to a single user or multiple users. However, interaction students and a teacher address a problem, the teacher cannot constantly online every time, and it is not possible for the teacher to deal with lots of question from students all the time and in a timely manner. In the collaborative learning, students are encouraged to ask question [7]. Therefore, there is a need to describe an automated Q and A system to support learning efficiency of collaborative learning.

In this paper, we proposed question answering system for an effective collaborative learning. With the proposed system, brings a new Q\&A system, student can attach their question when they want collaborative using collaborative learning capitalize on one another's resources and skills. Students can ask their questions to the group when they want to collaborate with others, asking one another for information, evaluating one another's ideas. The method also considers that students can communication through Q\&A interaction such a discussion forum to support information sharing. The paper is organized as follows. First is the motivation for the question and answering system. Section 2 presents the related works on collaborative learning. Section 3 explains about the question answering mechanism proposed. In section 4, present the architecture of system that we proposed an interactive effective Q\&A system for collaborative learning. The user interactions between user and the system are explained. Finally, section 6, are summary and conclusion of this paper.

\section{RELATED WORK}

Collaborative learning is one of the study groups. Some studies show that students get the most current learning through group rather than independently [8] [9]. Studies by the OTTER Group [10] have shown that the ideal class is organized around $50 / 50$ rule. At least $50 \%$ of the time students spend is spent interacting with and learning about the other student in the virtual classroom. The social aspect of the classroom is an important factor. If social aspect missing, than student dissatisfaction rises dramatically, as does the attrition rate. In this learning mode, which is collaborative learning, students who are interested in sharing their knowledge from a learning group to communicate and discuss all kinds of questions, asking one another for information, evaluating one another's 
idea for help and teach each other. Therefore, learning is both a group activity and a social process and thus learning performance is strongly affected peers [11].

In the development of networks, comprise all forms of electronically supported learning and teaching that can eliminate the obstacles of time and space. In the collaborative learning, students can take part by computer at anyplace, at the same time or different time (synchronous and asynchronous). Researchers have used activity theory to analyze Computer Supported Collaborative Work (CSCW) system [12]. Group communication relationship [13] refers to the intragroup relationships determined by the interactions among members. However, how to form a learning group after the group is a problem in collaborative learning.

Several study about Q\&A for collaborative learning had been done. An application of Question Answering System for Collaborative Learning has been designed. In this application learners can attach their question to the group when they want to collaborate with others, and the teacher providing answers to them. In this case, the collaborative between the students and the teacher to gain knowledge, and becomes question answering system like a virtual teacher [7] [14]. In this paper, we proposed question answering system for an effective collaborative learning. The originality of the system is bringing a new Q\&A system, in which students can attach their questions to the group when they want to gaining and sharing knowledge with others, by collaborative learning capitalize on one another's resources and skills (asking and evaluating one another's idea), then each of the answer will compare with encyclopedia data base.

\section{Proposed E-LEARning System With KNOWLEdGE- BASE SYSTEM}

E-learning system, which is proposed here is based on knowledge base system which allows acquires knowledge about students and uses the acquired knowledge. Once students learn with the proposed system through questions and answers, then all students can use the previously acquired Q\&A information which relates to the current question. Figure 1 shows the complete overview proposed of Question Answering Mechanism.

\section{A. Proposed Question Answering System}

An online Wikipedia-encyclopedia was chosen as the corpus for the task. Wikipedia is a free, web-based, collaborative, multilingual encyclopedia project supported by the non-profit Wikimedia Foundation. Indonesian Wikipedia is the edition of Wikipedia in the Indonesian language; there were over 100.000 articles in the Indonesian Wikipedia project. In the research proposed, we using Indonesian Q\&A system specifically in the Information Communication Technology Subject, for the Senior High School.

An automated Q\&A system in collaborative learning operates proposed work based on the question answering knowledge base. When a student needs some information, he or she can ask a question through a designed interface. When a new asked question enters the system, query is created. Then other students will response the question with answering and evaluating one another's ideas by vote. This representation is then compared with the representations of Wikipedia data base.

A similarity percentage is given between the student answer and any existing Wikipedia data base. Based on the biggest vote some time is not describing the best answer, after time for answering and voting finish, similarity percentage will be show for every answer. It aims to estimate whether which one of the answered considered with the Wikipedia data base. Also, student can access the topic question more clearly through the link provided. Such as [7], if no any information from Wikipedia comparing with neither student answer, nor the student is satisfied with any the answers (no proper match from the knowledge base), and then the teacher will answer or might be sent to the student. After the teacher manually answer the question, the new Q\&A set is formed and entered into the question answering knowledge base. When student meeting some difficulties or having no difficulties, a student can see what problems other students have encountered in learning now and in the past and see the answers or solutions the teacher offered by browsing the knowledge base. The whole process of question answering in the effective of collaborative learning is shown in figure 1.

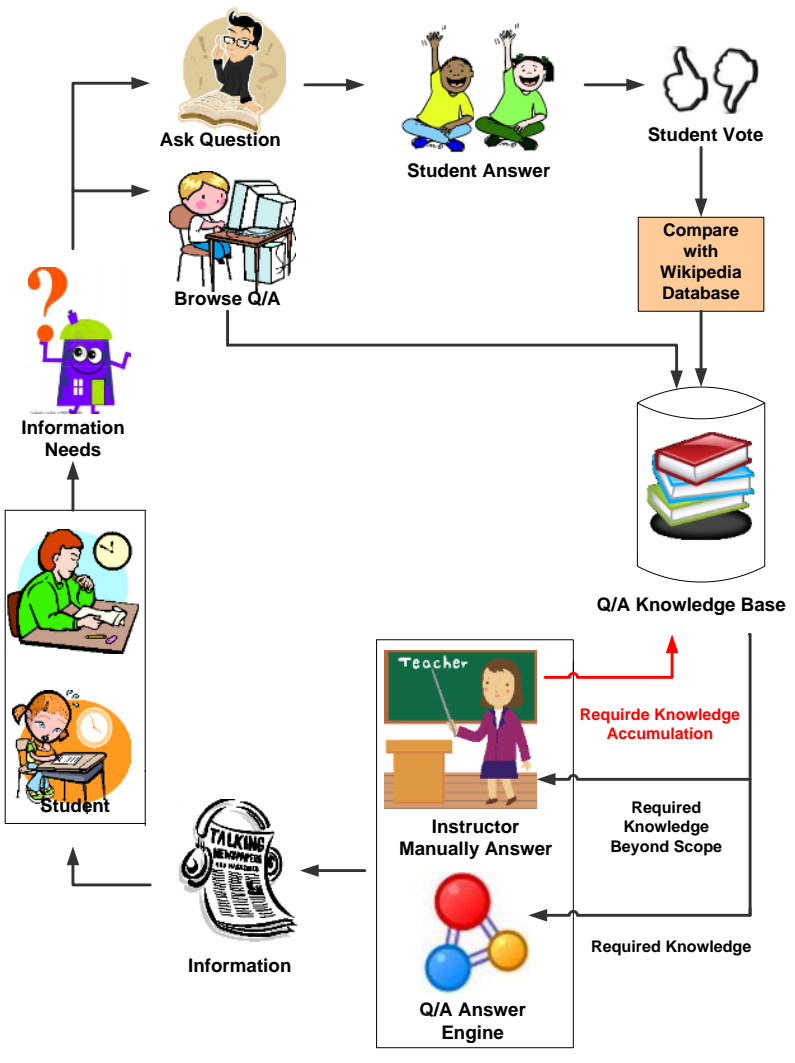

Figure 1. Question Answering Mechanism

\section{B. Architecture of Question and Answering System}

The architecture of the question answering system is shown in figure 2. There are eight main components in the system, including the student agent $\mathrm{Q}$, the student agent $\mathrm{A}$, question analysis and query generation, Q\&A acquirer, question answering knowledge base, similarity machine, Q\&A browsing component, answer generator. 


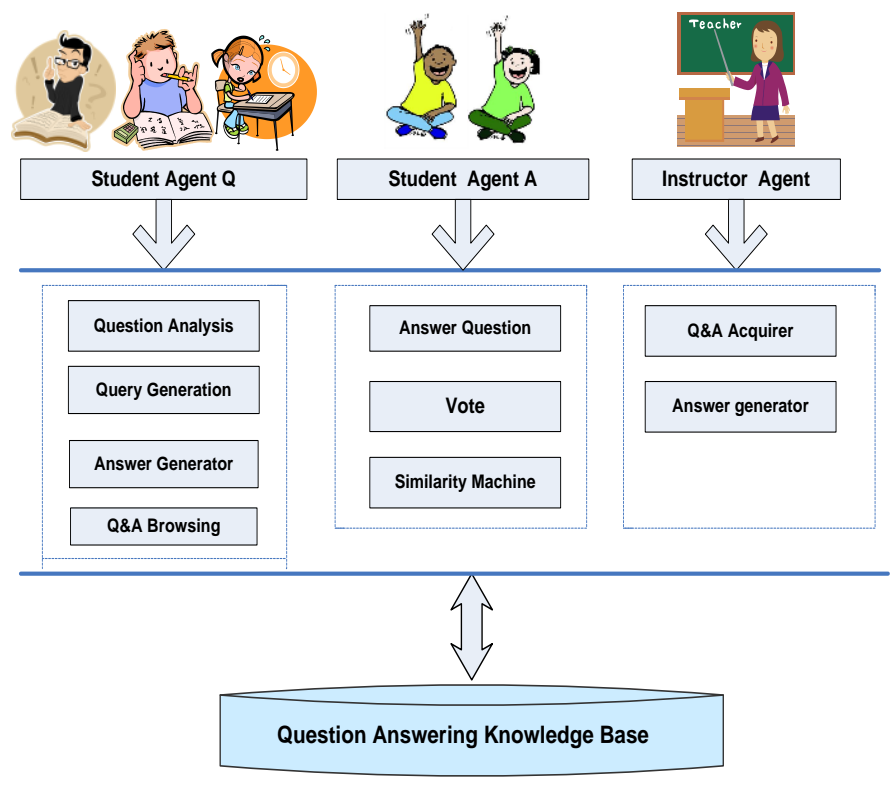

Figure 2. Architecture of Question Answering System

The functions of the components are briefly described as follows,

\section{- $\quad$ Student Agent Q}

Student agent Q is to be the interface between student and the system. Student can send his or her question and receive answers (feedback from other student) from this interface. Beside from student also provide feedback from the teacher for manually answering and question if the system are not satisfied.

\section{- $\quad$ Student Agent A}

Student agent $\mathrm{A}$ is the component interface between student and the system provides an answer and voting question. In this component also provide similarity machine to match student answer with Wikipedia database, to ensure the answer considering biggest vote not necessary correct answer.

- Question analysis, query generation

An analyzed question represents an asyntactic and semantic analysis of a question. It serves as an interface between the question analysis and query generation stages. A query is a search engine query generated at the query generation stage and executed at the search stage.

\section{- Q\&A Acquirer}

If there is an unanswered question, the questions will temporarily stores at this component. When the teacher gets online, and then will manually answer the question with an interface (Instructor Agent). This component are very important for a reason that student's question might be not answer by other student or student's answer cannot compare with Wikipedia database, cause the information not relevant. A new Q\&A will be formed and saved to the Q\&A knowledge base, after a question replied by the teacher.

- Question Answering Knowledge Base
A knowledge base provides a means for collected, organized, shared, searched and utilized information. Q\&A knowledge base is a knowledge base where questions corresponding with answer. The knowledge will be accumulated and rich, through the process of student question answer and manually teacher answer.

\section{- Q\&A Browsing Component}

Beside asking a question, when student meeting some difficulties or having no difficulties, a student can see what problems other students have encountered in learning now and in the past and see the answers or solutions the teacher offered by browsing the knowledge base.

\section{- Answer Generator}

Several interfaces have been designed, to let students and teacher is able to interact with the system. The interfaces for the student to ask question and get answers (Student Agent A) are developed as shown in Figure 3. Under the interface in figure 3, student can key in an Indonesian question and get an answer immediately without waiting for the teacher to get online. Then other student can answer the question and can discuss whether which one of their answer as same as their opinion. After that, Q\&A system makes a point for each of student answer that closely with Q\&A system answer. If no satisfactory answer is found, then it will present the teacher demanding a manual answer whenever he or she is online.

\section{- Similarity Machine}

This component uses Levenstein and Word similarity method to calculate the similarity between students answer and Wikipedia database. In the question analysis, extracting question query is necessary. Specific interrogation all has question query focus, usually question word $(\mathrm{Qw})$ is the question feature, such Qw as what, when, where, who, why and how. In this system we using what, when, where, who type of question. How and why, are notable mission because it's procedural answer. Using Levenstein for what question (Definition expectations), to calculate percentage Levenstein distance between two length string (students answer and the knowledge base answer). And using Word similarity for when, where, and who question. In the word similarity, the distance of words is a real number in $[0, \infty]$ a word and its own distance is zero. The following equation is word similarity formula.

$\operatorname{Sim}(W 1, W 2)=\frac{\propto}{\operatorname{Dis}(W 1, W s)+\propto}$

Where $\alpha$ is an adjustable parameter.

\section{User Interactions with Question and Answering System}

Several interfaces have been designed, to let students and teacher be able to interact with the system. The interfaces for the student to ask questions and get answers (Student Agent A) are developed as shown in figure 3 . Under the interface in figure 3, student can key in an Indonesian question and get answer immediately without waiting for the teacher to get online. Then other student can answer the question and can discuss whether which one of their answer as same as their opinion. After that, Q\&A system makes point for each of student answer that closely with Q\&A system answer. If no satisfactory answer is found, then student can press a button to 
send the question to the Q\&A acquirer, which will then present the answered questions to the teacher demanding for a manual answer whenever he is online.

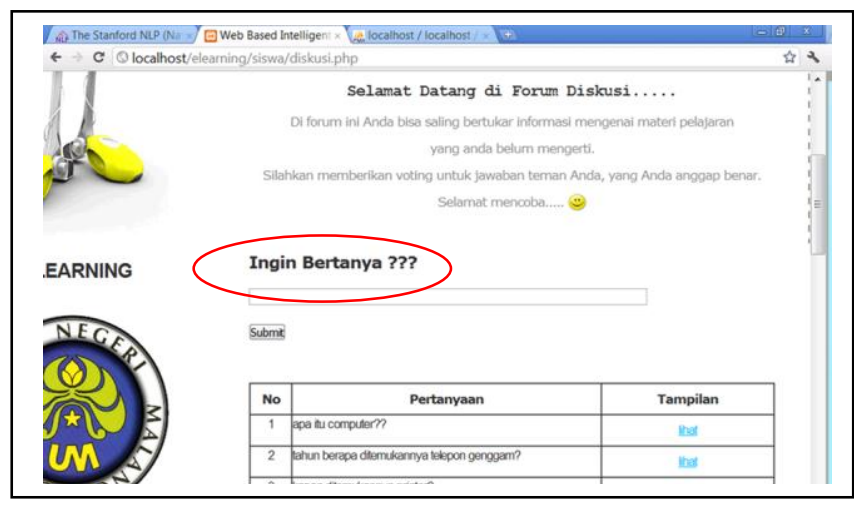

(a)

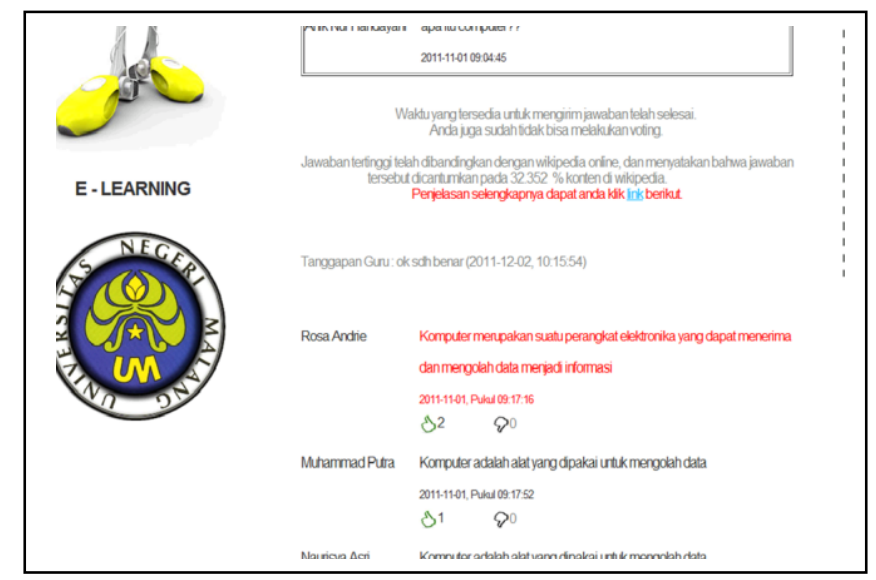

(b)

Figure 3. Student's interfaces question and get the answer

Beside asking a question, when student meeting some difficulties or having no difficulties, through the interfaces in figure 4, a student can see what problems other students have encountered in learning now and in the past and see the answers or solutions the teacher offered by browsing the knowledge base.

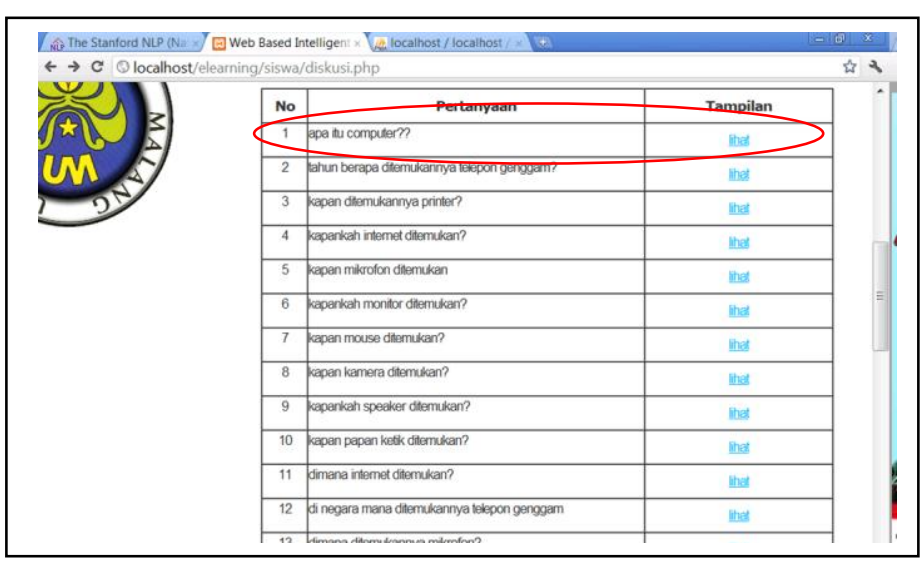

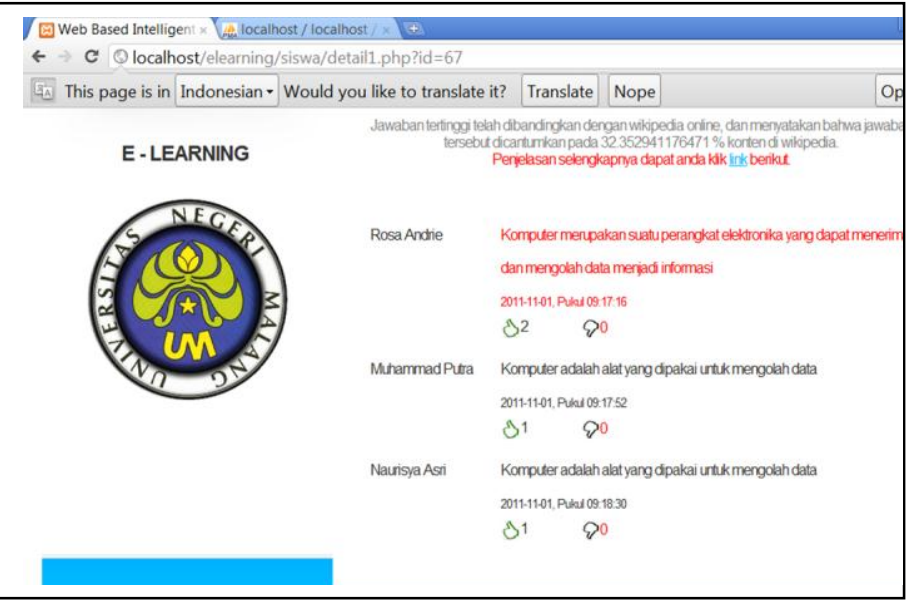

(b)

Figure 4. Browsing activities

\section{EXPERIMENT}

According to the method described above and the structure of question and answering system, we build an experimental system in Indonesian Q\&A system specifically in the Information Communication Technology Subject, for the Senior High School. We choose 40 questions, and there are two indexes in this experimental, precision and recall. The calculation formula is as follow:

precision $=\frac{a}{a+c} \times 100 \%$

recall $=\frac{a}{a+b} \times 100 \%$

In the formula, $a$ is the number of right matching of questions; $b$ is the number of without matching of question; $c$ is the numbers of wrong matching questions. Through the experiment, we can get the data of precision and recall, the result is in Table 1.

\section{TABLE $1 . \quad$ RESULT OF EXPERIMENT}

\begin{tabular}{|l|l|}
\hline Question & 40 \\
\hline Answer & 120 \\
\hline Right Matching & 57 \\
\hline Without Matching & 57 \\
\hline Wrong Matching & 6 \\
\hline Precision & $90,48 \%$ \\
\hline Recall & $50 \%$ \\
\hline
\end{tabular}

From the table, from the 40 question and 120 answer there are 57 answer are without matching because where the machine similarity get the keyword from the student question, there is not information about keyword question in the Wikipedia data based. In this case, the teacher will provide answers to students. After a question is replied by the teacher, not only the student will be notified, but also a new Q\&A set will be formed and saved to the question answering knowledge base. We have this component for a reason that student's questions might be unanswered by the system due to no suitable or desirable answers for them in the knowledge base. 


\section{CONCLUSION}

In this research, a system for online automatically answering students' questions in the collaborative learning environment has been designed. The system operated upon the question answering knowledge base. In the knowledge base, pairs of question with its corresponding answer (Q\&A sets) were collected through the process of students asking questions and other students will response the question with answering and evaluating one another's ideas by vote. This representation is then compared with the representations of Wikipedia data base. A similarity percentage is given between the student answer and any existing Wikipedia data based. Based on the biggest vote some time is not describe the best answer, after time for answering and voting finish, similarity percentage will show for every answer. It aims to the students who asked the question and information for students who answer and vote can estimate approximately correct answer. Also, students can access the topic question more clearly through the link provided. It was very important to have such a system in collaborative learning environment. It benefited both the teacher and the students; students can look for the answers to their questions without the constraint of time and space.

\section{REFERENCES}

[1] Dillenbourg, P. (1999). Collaborative Learning: Cognitive and Computational Approaches. Advances in Learning and Instruction Series. New York, NY: Elsevier Science, Inc.

[2] Chiu, M. M. (2000). Group problem solving processes: Social interactions and individual actions. Journal for the Theory of Social Behavior, 30, 1, 27-50.600-631.

[3] Chiu, M. M. (2008).Flowing toward correct contributions during groups' mathematics problem solving: A statistical discourse analysis. Journal of the Learning Sciences, 17 (3), 415 - 463

[4] Mitnik, R., Recabarren, M., Nussbaum, M., \& Soto, A. (2009). Collaborative Robotic Instruction: A Graph Teaching Experience. Computers \& Education, 53(2), 330-342.

[5] Chiu, M. M. (2008). Effects of argumentation on group micro-creativity. Contemporary Educational Psychology, 33, 383 - 402.

[6] Chen, G., \& Chiu, M. M. (2008). Online discussion processes. Computers and Education, 50, 678 - 692

[7] Wang, C.C., Hung J.C., Yang C.Y., Shih T.K. (2006). An Apllication of Question Answering System for Collaborative Learning. IEEE Conference on ICDCSW'06

[8] Johnson, D.W. and Johnson R.T. (1999) Cooperation and competition:
Theory and research. Edina. MN: Interaction Book Company

[9] Slavin, R. (1996) Research on cooperative learning and achievement: what we know, what we need to know. Contemporary Educational Pshchology, 21, 1, pp. 43-69.

[10] Gilrory, K.(2001). Collaborative e-learning: the right approach ([Online].Available at : http://www.ottergroup.com/otter-withcomments/right_approach.html).

[11] Lave, J., and Wenger, E. (1991) Situated Learning: Legitimate Peripheral Participation. Cambridge University Press, Cambridge

[12] Kuutti, K. (1991) The concept of activity as a basic unit of analysis for $\mathrm{CSCW}$ research. Proceedings of the Second European Conference on Computer-Supported Co-operative Work: EC-CSCW'91 (eds. L.J. Bannon, M. Robinson \&K. Schmidt) pp. 249-264, Kluwer, Dordrecht.

[13] Watzlawick, P. (1967) Pragmatics of Human Communications: A Study of Interactional Patterns. Pathologies and Paradoxes. W.W. Norton, New York.

[14] Bahreininejad A, Alinaghi Tanaz, 2011, A Multi Agent Question Answering System for E-Learning nad Collaborative Learning, nternational Journal of Distance Education Technologies, 9(2), 23-39, April-June 2011.

[15] Xu Jinzhong, Jia Keliang, Fu Jibin. 2008. Research of Automatic Answering System in Network Teaching. The 9th International Conference for Young Computer Scientist.

\section{AUTHORS PROFILE}

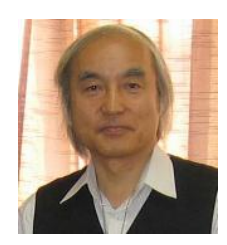

Kohei Arai received BS, MS and PhD degrees in 1972, 1974 and 1982, respectively. He was with The Institute for Industrial Science and Technology of the University of Tokyo from April 1974 to December 1978 and also was with National Space Development Agency of Japan from January, 1979 to March, 1990. During from 1985 to 1987, he was with Canada Centre for Remote Sensing as a Post Doctoral Fellow of National Science and Engineering Research Council of Canada.

He moved to Saga University as a Professor in Department of Information Science on April 1990. He was a councilor for the Aeronautics and Space related to the Technology Committee of the Ministry of Science and Technology during from 1998 to 2000 . He was a councilor of Saga University for 2002 and 2003. He also was an executive councilor for the Remote Sensing Society of Japan for 2003 to 2005. He is an Adjunct Professor of University of Arizona, USA since 1998. He also is Vice Chairman of the Commission A of ICSU/COSPAR since 2008. He wrote 26 books and published 227 journal papers.

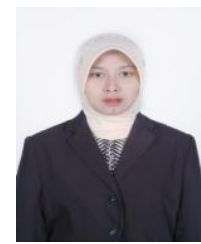

Anik Nur Handayani received the B.E. degree in electronics engineering from Brawijaya University, and the M.S. degree in Electrical Engineering, from Institute of Technology Sepuluh Nopember, Surabaya, Indonesia, in 2004 and 2008, respectively. She is currently a $\mathrm{PhD}$ Student at Information Science in Saga University, Japan. 